\title{
Acute renal infarction
}

\author{
Yasuharu Okuda $\cdot$ Raakhee Mahajan
}

Received: 7 April 2008 / Accepted: 30 July 2008 / Published online: 22 August 2008

(C) Springer-Verlag London Ltd 2008

A 58-year-old male presented with acute onset left-sided abdominal pain for 1 day. His pain was constant, aching, without any associated nausea or vomiting; his last bowel movement was the prior evening and normal. He denied fevers, chills, loss of appetite, and dysuria. Medical history was significant for hypertension. Physical exam revealed an irregular rhythm, left-sided mid-abdominal tenderness to deep palpation without rebound or guarding, and mild costovertebral-angle tenderness. EKG showed new onset atrial fibrillation. Urinalysis was negative. Subsequent contrast computed tomography of the abdomen and pelvis (Fig. 1) revealed the diagnosis.

The computed tomography image demonstrated multiple areas within the left kidney that did not enhance with contrast, which is consistent with acute renal infarction. The patient was anticoagulated with intravenous heparin, maintaining normal renal function through discharge. Renal infarction is a rare disorder typically affecting patients after the age of 60. Patients will often present with abdominal pain, hematuria, fever, nausea, vomiting, and/or oliguria and may have a history of atrial fibrillation. Laboratory findings can show hematuria on urinalysis and leukocytosis, elevated creatinine, and a markedly elevated LDH on serum analysis. Differential diagnosis includes diverticular disease, aortic aneurysm, gynecological disorder, and appendicitis.

Y. Okuda $\cdot$ R. Mahajan

Department of Emergency Medicine \& Medical Education,

Mount Sinai School of Medicine,

One Gustave L. Levy Place,

New York, NY 10029, USA

\section{Y. Okuda $(\square)$}

Department of Emergency Medicine,

Mount Sinai School of Medicine,

One Gustave L. Levy Place, Box 1149,

New York, NY 10029, USA

e-mail: yasuharu.okuda@mssm.edu

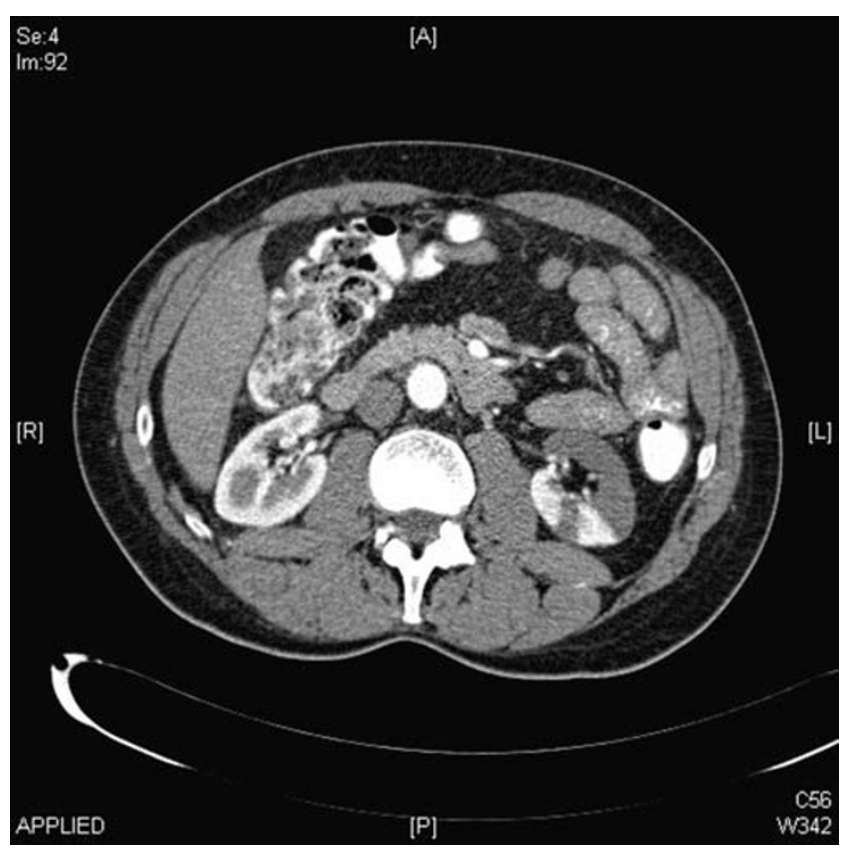

Fig. 1 Contrast computed tomography of the abdomen and pelvis

While renal angiography is the gold standard, CT abdomen with contrast is considered diagnostic for renal infarction. Standard and Doppler ultrasound, to date, is of limited value, though recent studies suggest a possible role for contrast-enhanced ultrasonography [1]. Late diagnosis does not preclude normal renal function.

\section{Reference}

1. Bertolotto M, Martegani A, Aiani L, Zappetti R, Cernic S, Cova MA (2008) Value of contrast-enhanced ultrasonography for detectin renal infarcts proven by contrast-enhanced CT. A feasibility study. Eur Radiol 18:376-383 